\title{
Subcellular features of calcium signalling in heart muscle: what do we learn?
}

\author{
Ernst Niggli and Peter Lipp
}

I $n$ this short review we discuss possible mechanisms for the regulation of calcium signalling in cardiac muscle on the subcellular level. We shall concentrate particularly on phenomena pertinent to the calcium induced calcium release (CICR) mechanism and to excitation-contraction coupling. Recently, subcellular spatial features and elementary events of the calcium signalling system have been revealed in heart muscle. and also in other cells with video imaging of fluorescent calcium indicators and confocal microscopy techniques. These exciting new findings will be discussed and a scheme of excitation-contraction coupling which involves the subcellular microarchitecture of this signalling network will be introduced. This model may also account for several apparently conflicting experimental results which have provoked stimulating discussion in the past. For a more general discussion of excitation-contraction coupling in cardiac (and skeletal) muscle the reader is referred to other recent reviews. ${ }^{-4}$

\section{Cells need signal transduction mechanisms}

Signal transduction across the cell membrane and within the cylosol represents a fundamental need of every living cell. A variety of transport mechanisms has evolved to overcome the diffusional barrier of the cell membrane which protects the cell interior but also separates it from the environment. Receptors, channels, and transporters have developed to convey information and metabolites across the cell boundary. Frequently, the intracellular signal is carried by a second messenger which is quite different from the extracellular signal. For example, many hormone receptors are coupled to the effector proteins through intracellular second messengers. Calcium ions are an almost ubiquitously used messenger and arc involved in intraccllular cvents as diverse as fertilisation, cell division and growth, secretion, and excitation-contraction coupling in muscle cells.

In principle, the information can be encoded in the signal in various ways in each of these processes. In biological systems two strategies are generally used: frequency modulation and amplitude modulation. Many signals show an all or none behaviour (for example, action potentials in excitable cells, some types of cytosolic calcium oscillations), which does not allow for encoding the information in the amplitude of the signal. In such systems, frequency modulation is used to grade the effect: the higher the frequency of the signals the larger is the resulting response. Examples are sensory neuronal input and the output from motor neurones to skeletal muscle cells. In signalling pathways that do not show all or none behaviour the response can be a continuous function of the signal strength and thus have a variable amplitude. Examples are receptor potentials from retinal and cochlear cells. Generally, signalling systems with a large positive feedback tend to behave in an all or none fashion while a graded response is the hallmark of negative or low positive feedback.

\section{The cardiac muscle cell: on the verge}

For a detailed understanding of cardiac excitationcontraction coupling several apparently contradictory experimental findings need to be reconciled with any comprehensive coupling model. Some of these experimental discrepancies may be related to uncertainties about the positive feedback inherent in the CICR mechanism. In cardiac muscle a transient increase of the intracellular calcium concentration represents the signal which links electrical excitation of the membrane to mechanical activity of the cell (see fig 1). In most species calcium release from the sarcoplasmic reticulum is the source of a large fraction of the total calcium required to initiate contraction. ${ }^{15}$ Calcium influx via voltage dependent calcium channels only serves as a trigger signal that is amplified severalfold by CICR. Since the output signal of the CICR results in an increase of cytosolic $\left[\mathrm{Ca}^{2+}\right]$ that is chemically identical to the trigger signal itself, this amplification system is expected to show a large positive feedback and therefore a tendency for all or none behaviour. Indeed, several recent experimental obscrvations provide support for this vicw. For example, propagating subcellular waves of calcium have been revealed in isolated cardiac myocytes by calcium imaging techniques. These calcium waves are believed to be actively driven by the CICR mechanism showing a large positive feedback. ${ }^{6-8}$ The presence of an all or none CICR would imply that the calcium release mechanism is always completely activated and cannot be modulated in these cells. Also, since the heart requires a fixed 1:1 coupling between electrical excitation (the action potential) and mechanical activity (the twitch contraction), direct frequency modulation of force cannot

University of Bern, Department of Physiology, Bühlplatz 5, 3012 Bern, Switzerland: E Niggli, P Lipp. Correspondence to Dr Niggli. 


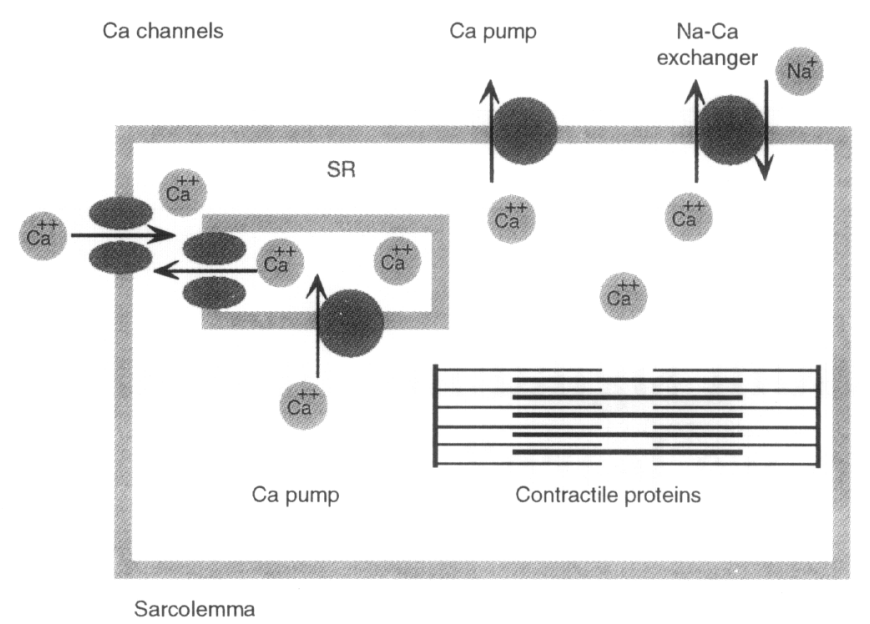

Figure 1 Common pool diagram of the events of cardiac excitation-contraction coupling. During the action potential, voltage dependent $\mathrm{L}$-type $\mathrm{Ca}^{2+}$ channels open and the resulting transsarcolemmal influx of $\mathrm{Ca}^{2+}$ triggers a larger $\mathrm{Ca}^{2+}$ release through the $\mathrm{Ca}^{2+}$ release channels of the sarcoplasmic reticulum $(\mathrm{SR})$. The increase in cytosolic $\left[\mathrm{Ca}^{2+}\right]$ activates the contractile proteins. $\mathrm{Ca}^{2+}$ is removed from the cytosol by reuptake into the $\mathrm{Ca}^{2+}$ store via a ATP dependent SR $\mathrm{Ca}^{2+}$ pump. In the steady state an amount of $\mathrm{Ca}^{2+}$ equivalent to the trigger signal must be extruded from the cell. This is accomplished predominantly by the $\mathrm{Na}-\mathrm{Ca}$ exchange (working in parallel with the ATP dependent $\mathrm{Ca}^{2+}$ pumps of the sarcolemma).

occur in cardiac muscle, in contrast to the tetanic behaviour of skeletal muscle. Thus the question arises: is there any modulation of CICR in heart muscle whatsoever?

There are several lines of experimental evidence indicating that this is indeed the case. Various studies have suggested quite a low positive feedback in cardiac CICR. For example, in voltage clamp and fluorescent indicator studies calcium release has been shown to be approximately proportional to the calcium current over a wide range of voltages (for example see ${ }^{19-13}$ ), indicating the presence of amplitude modulation. More recently, sustained ryanodine sensitive calcium release from the sarcoplasmic reticulum was found at the threshold of activation of L-type calcium current. ${ }^{14}$ This type of release, as well as the release during a larger voltage clamp step, could be immediately interrupted by terminating the influx of calcium through L-type calcium channels, ${ }^{15}$ suggesting that CICR is under direct and continuous control of calcium influx and thus not activated in an all or none fashion. In another study using photolysis of caged calcium to provide the trigger signal for CICR, cell shortening was found to be graded with the amount of flash energy used. ${ }^{16}$ Experimentally, localised calcium transients were produced by only applying extracellular calcium to one edge of a single cardiac myocyte or by localised photolysis of caged calcium. ${ }^{17}$ Both approaches yielded calcium transients in a subcellular region of the cell that, surprisingly, did not propagate as calcium waves, again suggesting that the positive feedback in CICR has to be quite low. Taken together, these results indicate that some amplitude modulation of the CICR is possible in cardiac muscle cells and that the positive feedback of CICR has to be rather low under some conditions.

What can we conclude from these results? How can we reconcile these discrepancies with present models of cardiac excitation-contraction coupling and CICR? Some studies seem to show that the positive feedback in CICR is high while others indicate that the feedback is low. But it is not clear whether the divergent conclusions are unquestionable or depend significantly on differences in the experimental approach or the conditions of each study. Nevertheless, we may assume as a working hypothesis that the amount of positive feedback of CICR is somehow variable in cardiac muscle. This feature of CICR would allow us to account for most experimental findings outlined above. But with this hypothesis the question still remains open as to how this variability is achieved by the individual cell. At least a partial answer may lie in the elementary nature of the calcium signalling process which has only recently become spatially resolvable.

\section{Confocal microscopy reveals subcellular spatial detail of CICR}

Significant additional insight into the subcellular calcium signalling mechanisms resulted from the use of fluorescence imaging and confocal microscopy techniques. Confocal imaging of calcium sensitive fluorescent indicators greatly improves the subcellular spatial resolution because the blurring effect of out of focus fluorescence can be removed. ${ }^{18-20}$ Using this "optical sectioning" technique we recently made an unexpected observation in neonatal rat myocytes kept in primary culture. $\Lambda \mathrm{fter}$ loading these cells with the calcium indicator fluo-3, spontaneous calcium release events could be detected with the confocal microscope, even under conditions not believed to induce cellular calcium overload. ${ }^{21}$ Analysis of the spontaneous release events revealed three distinct patterns of calcium release: (1) a sudden increase of intracellular $\left[\mathrm{Ca}^{2+}\right]$ which was homogeneous throughout the cell and closely resembled a calcium transient induced by a voltage clamp depolarisation; this type of calcium release most probably arose from a spontaneous action potential of the cell; (2) a subcellularly localised spontaneous release that subsequently propagated throughout the cell as a calcium wave; this release pattern corresponded to the calcium waves described in adult cardiac myocytes (see ${ }^{6-8}$ and fig 2 ) and suggested that the positive feedback of CICR may be large in these

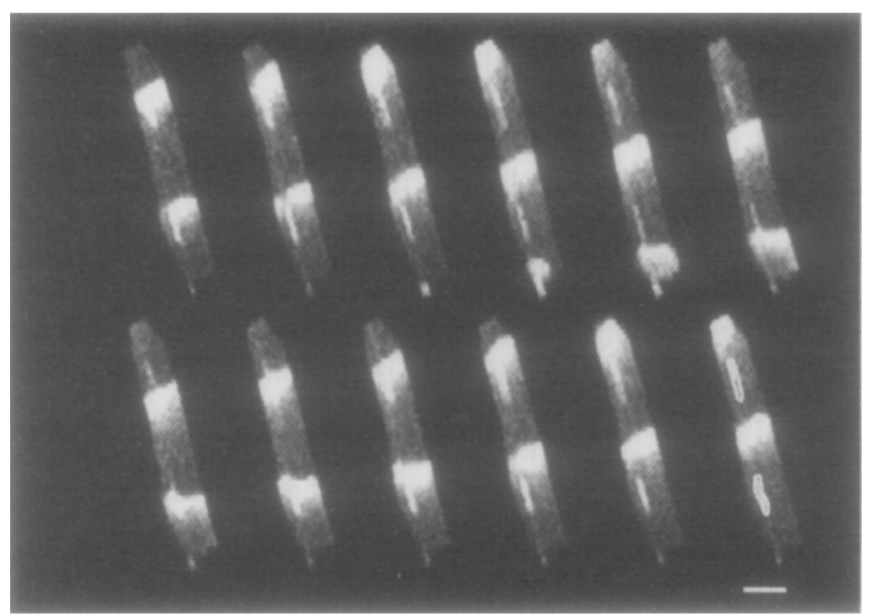

Figure 2 Linear waves of $\mathrm{Ca}^{2+}$ in a guinea pig ventricular cell. This panel shows a sequence of ratiometric confocal sections scanned at intervals of $125 \mathrm{~ms}$. Fluorescence ratios were obtained after loading the cell with a mixture of the two $\mathrm{Ca}^{2+}$ indicators fuo3 and fura-red. Spontaneous $\mathrm{Ca}^{2+}$ release initiated as a localised $\mathrm{Ca}^{2+}$ release event in the lower left region of the cell and subsequently spread as a $\mathrm{Ca}^{2+}$ wave along the entire cell. The propagation velocity of the wave was estimated to be $65 \mu \mathrm{m} \cdot \mathrm{s}^{-1}$. The two nuclei of the myocyte are outlined in the last frame. The scale bar corresponds to $20 \mu \mathrm{m}$. Reproduced from reference 25 . 


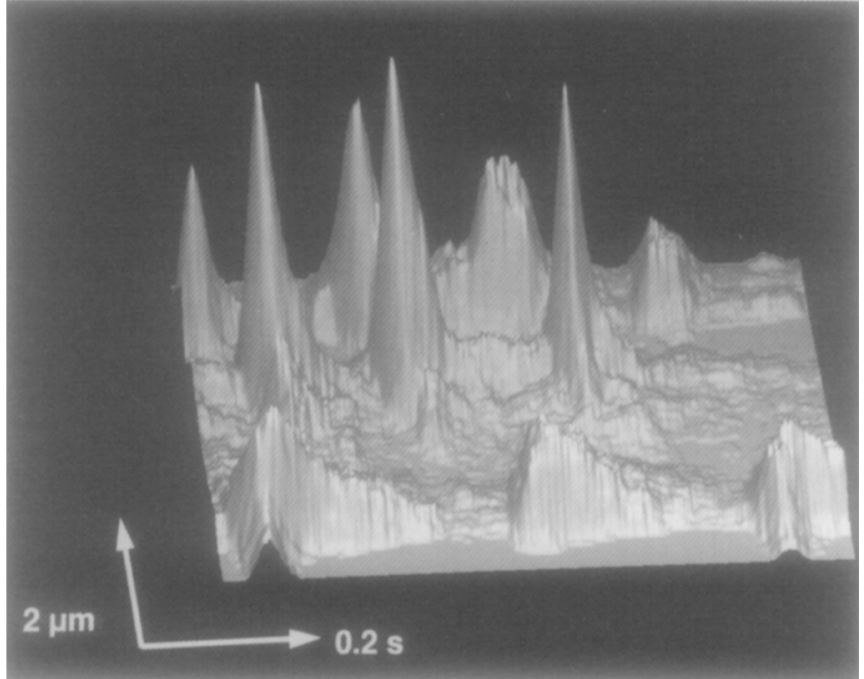

Figure 3 Subcellularly localised spontaneous $\mathrm{Ca}^{2+}$ release events without propagation. A confocal line scan of fluo-3 fuorescence was converted into a surface plot to visualise the $\mathrm{Ca}^{2+}$ signals in the scanned line versus time. Localised release events occur at apparently random times and at several locations along the scanned line. It is obvious that the $\mathrm{Ca}^{2+}$ signals do not propagate in space (that is, in the spatial dimension of the surface plot running towards or away from the viewer). Reproduced from reference 21 .

cells; (3) the most surprising finding was a subcellular focal calcium release that remained local and did not propagate noticeably, indicating that the positive feedback of CICR is small in cells showing this type of calcium signalling pattern (see fig 3 ). Since this type of spontaneous calcium release was seen quite frequently in the same culture as the wavetype release we concluded that the positive feedback of calcium signalling has to be quite variable from cell to cell. The variability was initially believed to be related to morphological (that is, developmental) peculiarities of the sarcoplasmic reticulum in individual cells. This view was conceivable because it is known that the sarcoplasmic reticulum and the excitation-contraction coupling machinery undergo major developmental changes in neonatal rat cardiac myocytes. ${ }^{22}$

The striking difference in the calcium signalling behaviour may, however, also result from some unknown functional differences that change with time within individual cells. We considered this possibility to be more likely after the observation of spontaneous transitions between the different calcium release patterns in individual cells. ${ }^{21}$ In these cases, a period of spatially random focal release events without noticeable propagation was interrupted by a calcium wave propagating along the entire cell. The wave itself was followed by a refractory period during which no spontaneous release event could be detected. Recently, localised calcium release events with similar properties (calcium "sparks") were also reported in freshly isolated adult rat cardiac myocytes. ${ }^{23}{ }^{24}$ The presence of several subcellular calcium release patterns is thus not a peculiarity of neonatal cells or cells in culture.

We also observed calcium signalling features that strongly suggest a variability in the degree of positive feedback in adjacent subcellular regions even within single adult cardiac myocytes isolated from guinea pigs. ${ }^{25}$ Although spontaneous focal calcium release events appear to be much less frequent in this species, planar calcium waves are known also to develop in guinea pig cardiac myocytes under conditions of calcium overload and they are believed to be driven by the autoregenerative CICR mechanism (see fig 2). These waves were usually triggered by a focal release event and travel along a linear path and at an almost constant velocity, usually ranging from 50 to $120 \mu \mathrm{m} \cdot \mathrm{s}^{-1} .{ }^{7826}$ Unexpectedly, we also found calcium waves that showed a path strikingly different from the simple linear route. As shown in fig 4 , spiral waves of calcium were observed to spin around the nucleus, a structure which does not actively participate in CICR and thus represents an obstacle for the calcium wave. Nevertheless, calcium could still enter and leave the nuclei slowly, presumably,by diffusion through the nuclear pores. Somctimes, cytosolic calcium waves only propagated on one side of the nucleus, the other side of the cell appearing to show a low positive feedback, resulting in a subcellular "conduction block". This conduction block then made it possible for the same wave to return on the other side of the nucleus, giving rise to a "micro reentry" phenomenon which could initiate and sustain the generation of a spiral wave. These results suggest that some regions of the cell could not easily be entered by the calcium wave, probably because they were in a state of lower positive feedback than other regions.

In various excitable media it has been observed that spiral waves which develop near the boundary with an inexcitable region show a characteristic behaviour: the spiral cannot enter this low gain zone but interacts with it in a way that leads to a shift of the spiral core along the boundary. ${ }^{27} \mathrm{We}$ have observed exactly this behaviour for calcium spiral

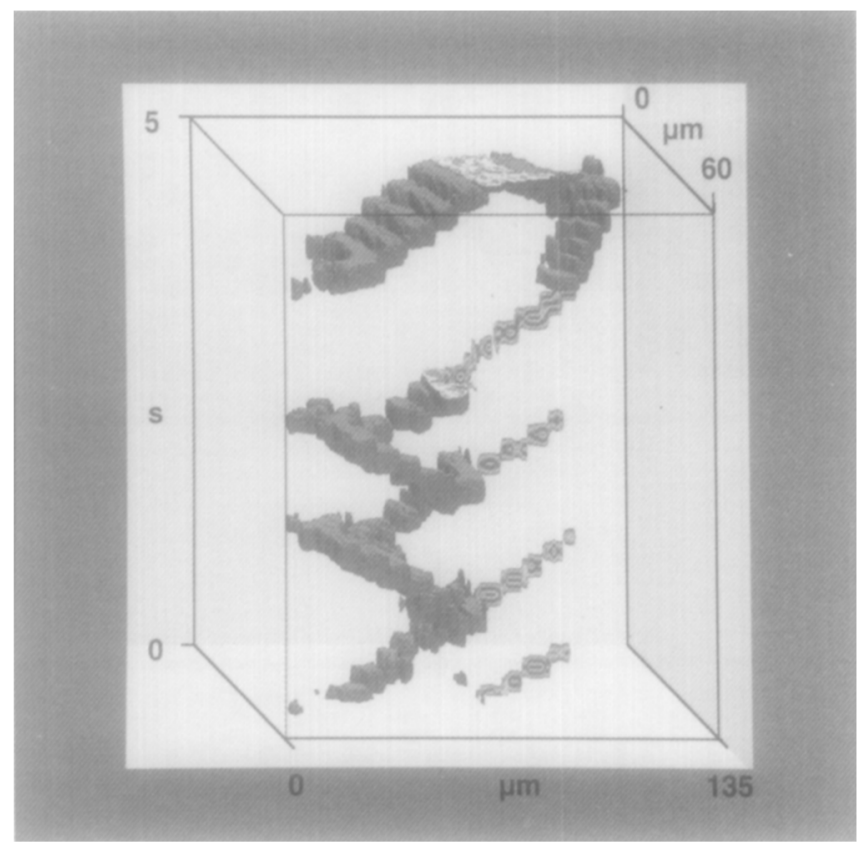

Figure 4 Pseudo three dimensional data stack illustrating spiral waves of $\mathrm{Ca}^{2+}$ and wave annihilation. A sequence of (two dimensional) confocal optical sections was converted into a data stack with the time running from the bottom to the top. After image processing with volume rendering software all regions with raised $\mathrm{Ca}^{2+}$ are shown as dark objects in space while regions with low $\mathrm{Ca}^{2+}$ remained transparent. $\mathrm{A} \mathrm{Ca}^{2+}$ wave travelling around a nucleus of the cardiac myocyte shows up as a corkscrew-like structure in the lower left part of the data cube. Linear $\mathrm{Ca}^{2+}$ waves propagate along the longitudinal cell axis at regular intervals and are shown as (blue) rods emanating from the spiral to the right. Above the spiral two $\mathrm{Ca}^{2+}$ waves travel in opposite direction and finally collide head on, thereby annihilating each other. Reproduced from reference 25. 
waves in cardiac myocytes. ${ }^{25}$ This finding again suggests the existence of subcellular regions showing low positive feedback, while other regions at the same time have a larger positive feedback and maintain the calcium wave.

\section{The sarcoplasmic reticulum of cardiac myocytes is a three dimensional network of subcellular elements}

The variety of observed calcium signalling patterns represents evidence that the sarcoplasmic reticulum is not a functionally homogeneous structure on the subcellular level. Besides low gain regions expected on morphological grounds (for cxample, the nuclei, mitochondria), larger areas within the cytosol appeared to show a below average gain. We therefore propose that the sarcoplasmic reticulum forms a three dimensional distributed network of functional elements. The morphological equivalent of these elements is not yet clear, but on functional grounds they manifest themselves as focal calcium release events without propagation. It is also not yet clear how many release channels actually contribute to a single release element. In this respect, a direct comparison of the kinetics of elementary release elements with the kinetics of calcium release channels is hampered by dramatically different properties of the channel in an intact cell compared to behaviour in the magnesium-free and ATP-free environment of the bilayer experiments.

A
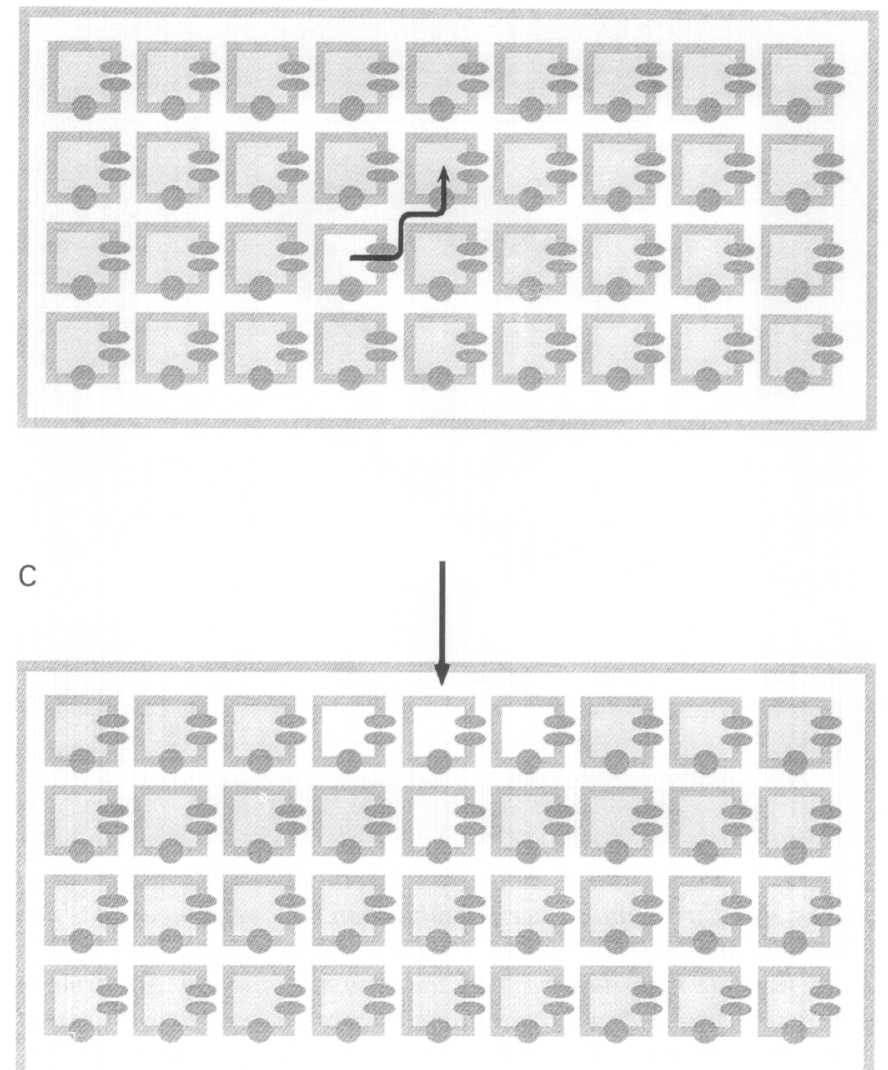

The individual sarcoplasmic reticular elements are independent from their neighbours to a certain degree, but are also partially coupled through the level and changes of the cytosolic calcium concentration in their vicinity. Each element contains processes that add to the positive feedback in the CICR (for example the sarcoplasmic reticular calcium release channels), but also has the means for a certain degree of negative feedback by removing calcium from the cytosol (that is, the sarcoplasmic reticular calcium pump). Occasional spontaneous openings of sarcoplasmic reticular calcium release channels are expected to occur because the open probability is never zero, even at physiological resting $\left[\mathrm{Ca}^{2+}\right]$. This stochastic opening of a single calcium release channel may then trigger additional calcium release channels of the same release element (due to the local increase in $\left[\mathrm{Ca}^{2+}\right]$ ). After such a spontaneous release event of a given sarcoplasmic reticular element two different scenarios can be imagined, as follows.

(1) When the neighbouring elements in the sarcoplasmic reticular calcium signalling network show a low positive feedback, the spurious release by one single element may not be sufficient to trigger additional calcium release from neighbours (see fig $5 \mathrm{~A}$ ). The calcium that was released accidentally would then be removed from the cytosol before it can do any harm. Otherwise, these small release events would be amplified by CICR and the resulting rise in $\left[\mathrm{Ca}^{2+}\right]$ would activate transient inward currents (for example, $I_{t i}$,
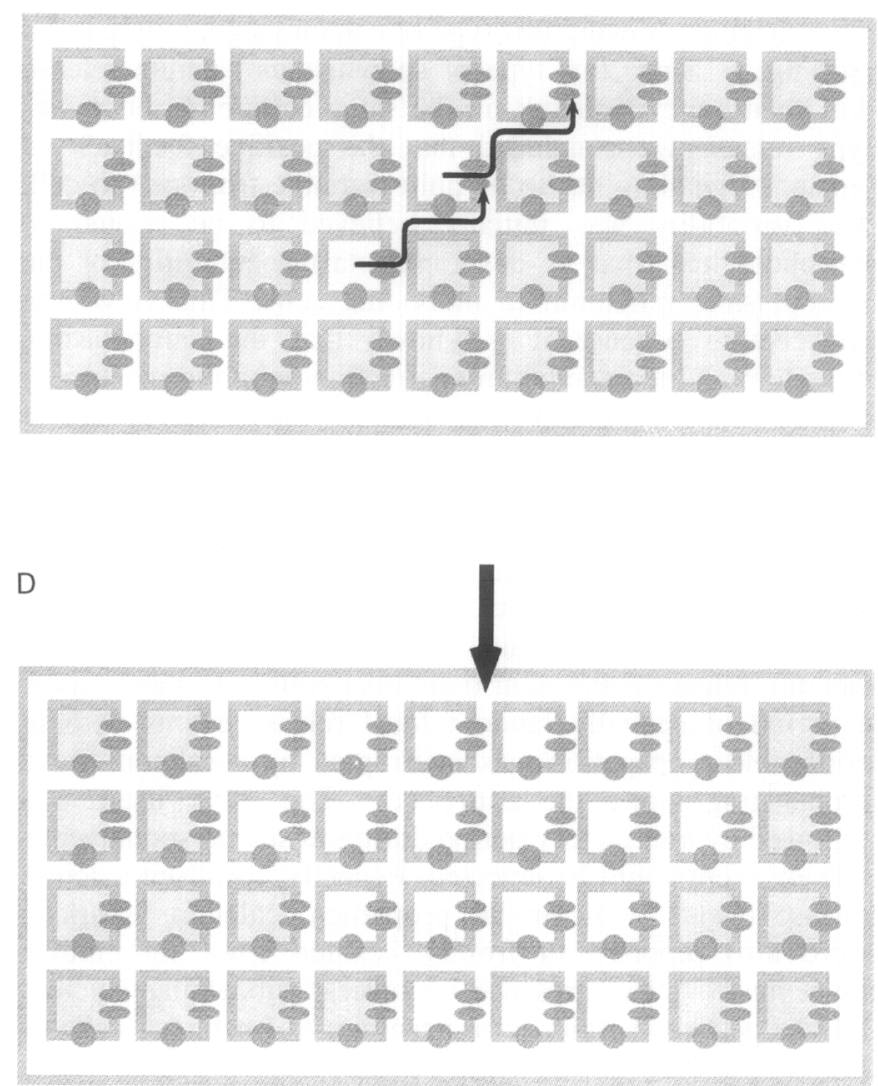

Figure 5 Diagram of a network model of the $\mathrm{Ca}^{2+}$ release mechanism in cardiac myocytes. (A) The sarcoplasmic reticulum consists of many individual $\mathrm{Ca}^{2+}$ release elements containing $\mathrm{Ca}^{2+}$ release channels and $\mathrm{Ca}^{2+}$ pumps. Accidental spontaneous release results in a localised release event followed by reuptake into neighbouring release elements via the $\mathrm{Ca}^{2+}$ pump. $(B)$ When the release is sufficiently large or when the trigger for release in neighbouring elements is low the focal release may initiate a propagating $\mathrm{Ca}^{2+}$ wave. $(C)$ With a network model consisting of many independent elements a small $\mathrm{Ca}^{2+}$ influx across the sarcolemma may trigger $\mathrm{Ca}^{2+}$ release from only few elements located in the vicinity of the cell membrane. $(D)$ A large $\mathrm{Ca}^{2+}$ influx will tend to trigger more $\mathrm{Ca}^{2+}$ release elements by means of a recruitment process. 
$\mathbf{I}_{\mathrm{NaCa}}$ ). Transient inward currents have been implicated in the generation of afterdepolarisations and cardiac arrhythmias. ${ }^{28}{ }^{29}$ A CICR mechanism with a certain degree of negative fecdback and the resulting threshold for amplification thus provides the necessary safety mechanism to prevent accidental action potentials from being triggered by the stochastic openings of single calcium release channels in vivo.

(2) After certain spontaneous release events a dramatically different scenario can also be observed. Occasionally, a localised spontaneous event is able to trigger additional calcium release from neighbouring sarcoplasmic reticular elements, giving rise to a diffusion reaction wave propagating over a limited subcellular distance or even throughout the entire cell (see fig 5B). In these cases, the delicate balance between the mechanisms providing some negative feedback and those responsible for positive feedback in CICR has changed. This change of overall gain may result in a different threshold for calcium release from the sarcoplasmic reticulum and lead to propagating calcium waves driven by the CICR mechanism. The observation of subcellular regions which do not allow the propagation of a calcium wave (resulting in a "subcellular conduction block") suggests that the individual elements of the sarcoplasmic reticular network can show significant differences in positive feedback, even within a single cell and at any given moment in time.

\section{Biochemical mechanisms underlying feedback modulation are largely unknown}

At present, we do not yet understand the biochemical basis for the differences in feedback among individual sarcoplasmic reticular elements. However, results obtained in various experimental systems provide some clues how a modulation of sarcoplasmic reticular gain might be accomplished. Even in early reports of wave-like activity and afterdepolarisations it was noted that an increase of the calcium load somehow increased the frequency of these events. ${ }^{28-30}$ More recent studies with fluorescent imaging systems now seem to indicate that the cytosolic resting $\left[\mathrm{Ca}^{2+}\right]$ does not change detectably despite a significant calcium overload of the sarcoplasmic reticulum..$^{31}$ In rat ventricular myocytes that were briefly treated with ouabain, the resting $\left[\mathrm{Ca}^{2+}\right]$ did not yet increase noticeably, while the triggered calcium transients were much larger than in control conditions and the calcium wave propagation was enhanced. Apparently, the sarcoplasmic reticulum contained more releasable calcium as a consequence of the calcium overload. This finding suggests that the calcium concentration in the lumen of the sarcoplasmic reticulum may play an important modulatory role in the CICR. In line with this hypothesis, an increase in the open probability of sarcoplasmic reticular calcium release channels incorporated into lipid bilayers has been observed after raising $\left[\mathrm{Ca}^{2+}\right]$ on the luminal side. ${ }^{32} 33$ The effect of intraluminal calcium on the open probability (or cytosolic calcium sensitivity) of the sarcoplasmic reticular calcium channel does not necessarily need to be a direct one. Allosteric interactions have been proposed to be important between the sarcoplasmic reticular calcium binding protein calsequestrin, the sarcoplasmic reticular protein triadin, and the calcium release channel. ${ }^{34}{ }^{35}$ Other biochemical mechanisms and sarcoplasmic reticular proteins may play modulatory roles as well, for example calmodulin, the $60 \mathrm{kDa}$ protein, or the FK506 binding protein (see ${ }^{32} 36$ for recent reviews). Other possibilities are phosphorylation and dephosphorylation reactions of the sarcoplasmic reticular calcium release channel protein ${ }^{37} 38$ and cyclic ADP ribose, an important messenger in the generation of the calcium fertilisation wave in sea urchin $\operatorname{eggs}^{39}$ which has been found to stimulate CICR in vesicles obtained from cardiac tissue, presumably by increasing the open probability of the calcium release channel. ${ }^{40}$

Other changes of the overall feedback in a given sarcoplasmic reticular element that do not require a modification of calcium release channel properties are possible. As we mentioned above, each sarcoplasmic reticular element also has mechanisms contributing some negative feedback to the system, the calcium pumps. When the function of calcium pumps providing negative feedback is impaired by back-inhibition mediated by the calcium load of the sarcoplasmic reticulum, the overall positive feedback of the sarcoplasmic reticular element will also increase. The calcium pumps themselves are also known to be modulated in cardiac muscle by phosphorylation of the associated protein phospholamban. ${ }^{41}$

Taken together, the biochemical basis for the observed subcellular heterogeneity in intact cells is unclear at present. It will be experimentally challenging to investigate these qucstions since in intact preparations many variables cannot easily be controlled and separated. The reductionist approach also faces difficulties since many biochemical interactions within this calcium signalling system may be altered or lost when studies are performed on the level of single molecules.

\section{Elementary calcium release events are also relevant for excitation-contraction coupling}

Is the elementary nature of cardiac calcium release only important to explain the mechanism of calcium waves or can we also learn something relevant for calcium signals during excitation-contraction coupling? We believe that the latter is indeed the case. As outlined above, several apparently controversial results exist regarding the intrinsic positive feedback in cardiac calcium signalling. The observation of a calcium release that is somehow graded with the amount of calcium current cannot easily be reconciled with the observation of propagating calcium waves driven by the same calcium release mechanism. Provided the sarcoplasmic reticulum is a three dimensional network of release elements, subcellular concentration gradients may allow for a modulation of calcium release during calcium signalling. For example, during the influx of calcium through L-type calcium channels the calcium concentration under the sarcolemma is expected to rise much higher than the bulk $\left[\mathrm{Ca}^{2+}\right]$ in the cytosol, especially in the limited space where the $\mathrm{T}$ tubular membrane is in close proximity to the sarcoplasmic reticular membrane (that is, diadic junctions). Sarcoplasmic reticular calcium release elements located close to the T tubular membrane may "see" a much higher $\left[\mathrm{Ca}^{2+}\right]$ than elements located further away from the surface. These elements may therefore also show a high apparent gain, as was proposed in a flash photolysis study on CICR in cardiac myocytes ${ }^{16}$ and in crayfish skeletal muscle cells. ${ }^{42}$ The obvious advantage of such a complex subcellular network is the capacity to be modulated in amplitude by a recruitment process in which fewer or more sarcoplasmic reticular calcium release elements are employed, depending on the magnitude of transsarcolemmal calcium influx (see fig 5, panels C and D). A recent experimental observation was interpreted as directly visualising this recruitment 
process in adult rat ventricular myocytes. When excitationcontraction coupling was partially uncoupled by reducing the L-type calcium current with a low concentration of nifedipine, spatial inhomogeneities of the calcium signal were revealed that were reminiscent of elementary calcium release events. ${ }^{43}$ Identical spatial patterns have also been observed under voltage clamp conditions when small calcium currents were induced by small depolarisations. ${ }^{12}$ However, when calcium currents of similar amplitude were activated by large depolarisations the spatial organisation of the calcium transients into elements was reduced.

Along the same line of reasoning, voltage dependent variations in gain have recently been explained by a local control of the calcium release elements by the sarcolemmal calcium influx. ${ }^{13}$ Let us compare two different calcium currents of identical peak amplitude, one on the ascending limb and the other on the descending limb of the calcium current-voltage relationship. The comparison of the two calcium currents reveals a fundamental difference on the single channel current level. At more negative potentials, the single channel current is comparatively large (due to the large driving force for calcium into the cell) but the open probability is small. At more positive potentials at an identical macroscopic calcium current the opposite is true. The open probability is higher but the single channel current is correspondingly smaller. Therefore, larger microdomains of increased $\left[\mathrm{Ca}^{2+}\right]$ around the L-type calcium channels are generated at more negative membrane potentials, thereby increasing the apparent gain. This notion also implies independence of sarcoplasmic reticular calcium release elements and is consistent with mathematical local control models of cardiac excitation-contraction coupling, ${ }^{44}$ where the calcium influx was proposed to be directly and locally responsible for the calcium release. Indeed, it has turned out to be impossible to model the amplification of the calcium signal by a CICR mathematically with common pool models, that is, with schemes where the entire sarcoplasmic reticulum has free access to a single pool of cytosolic calcium (see fig 1). However, other mathematical schemes called "cluster bomb" models have been able to account for many experimental findings mentioned above. In essence, local control, cluster bomb, and network models represent equivalent descriptions of the same signalling system - just the point of view is different: functional and spacial versus mathematical.

Local control in a signalling network may be complemented by "adaptation" of single calcium release channels to provide graded calcium release from the sarcoplasmic reticulum. Adaptation is a process by which calcium release channels reduce the open probability in the presence of increased calcium concentrations without being inactivated $^{45}$ (that is, the channels remain available for activation by a second, although larger, stimulus). Adaptation may underlie the inactivation process of CICR reported in skinned cardiac muscle cells ${ }^{46}$ and allow for graded calcium release.

\section{Submicroscopic concentration gradients}

These considerations ultimately also imply the existence of spatial gradients that have not yet been resolved with fluorescent imaging methods. However, a substantial body of indirect evidence has accumulated suggesting that submicroscopic concentration gradients are also important for our understanding of subcellular signalling mechanisms. ${ }^{47}$ For example, a suhsarcolemmal increase of the local sodium concentration has been proposed to explain the phenomenon of sodium current induced calcium release in cardiac myocytes. $^{48-51}$ By using subcellularly targeted fluorescent indicators it may soon become possible to localise concentration gradients that are on a submicroscopic scale. For example, targeted recombinant aequorins have been engineered to detect calcium signals from various organelles including the endoplasmatic reticulum, the cellular nuclei, and the mitochondria. ${ }^{52}$ In yet another approach, the fluorescent calcium indicator fura-2 was coupled to a lipophilic molecule that partitions into the membranes of living cells. Using this anchoring mechanism, the fluorophore becomes located primarily in the immediate vicinity of the membrane and thus reports the local calcium concentration with molecular spatial resolution. ${ }^{53}$ Indeed, both larger and faster calcium transients than with the regular fura- 2 were recorded with this technique in smooth muscle cells.

\section{Functional role of spatial gradients in other comparable signalling systems}

The question arises whether the features of calcium signalling and CICR found in cardiac tissue are also relevant to other cells using calcium signalling mechanisms for signal transduction. Indeed, recent reports show a remarkable similarity of calcium signalling systems from a variety of cells and with quite different molecular machineries. Oscillatory phenomena and calcium waves have been observed in many different cell types including smooth muscle cells, ${ }^{54}$ astrocytes, ${ }^{55}$ hepatocytes, ${ }^{56.57}$ and Xenopus oocytes. ${ }^{58}$ We can only mention a few examples in this review. Many cell types use a combination of the second messenger $\mathrm{IP}_{3}$ and calcium for signalling purposes. In these systems, an increase of the $\mathrm{IP}_{3}$ concentration represents the initial stimulus for calcium release from $\mathrm{IP}_{3}$ sensitive calcium stores. In contrast to the CICR mechanism, the trigger signal is thus chemically different from the output signal of the amplification process, although several feedback loops mediating an interaction between calcium and $\mathrm{IP}_{3}$ have been revealed. Questions related to the modulation of $\mathrm{IP}_{3}$ induced calcium signals have frequently been very similar to those relevant for CICR, and the two fields may learn more from each other than previously thought. At present, several key properties of the elementary CICR network are still unknown. Most interestingly, it is not yet clear how exactly the modulation by a recruitment process works. In principle, a recruitment could work with each single release element behaving in an all or none fashion or with each element capable of generating a graded response by itself.

This question can be addressed with fewer difficulties in systems with a trigger signal biochemically different from the output signal. In a recent study, the dose-response relationship for photorelease of $\mathrm{IP}_{3}$ from "caged" $\mathrm{IP}_{3}$ in Xenopus oocytes was compared for two illumination techniques. ${ }^{59}$ When the entire cell was illuminated, a graded dose-response was found. However, when significant photolysis was largely confined to a diffraction limited spot with a confocal illumination arrangement, the calcium signal was found to depend on the amount of UV light in an all or none fashion. This finding suggests that an individual calcium release element indeed behaves in an all or none fashion in these cells, while the graded response of the whole cell is due to a recruitment process.

Also in Xenopus oocytes, large spiral waves of calcium with properties similar to those we found in cardiac 
myocytes were described recently. ${ }^{58}$ In this system it was possible to test directly some hypotheses of feedback modulation by functionally expressing avian sarcoplasmic reticular calcium pumps at a high activity. This modification of the calcium release resulted in an increased frequency of individual calcium waves and narrowed their width. ${ }^{60}$ When the calcium influx into the oocytes was enhanced by hyperpolarisation, an acceleration of the wave propagation was found. ${ }^{61}$

\section{What to expect in the near future?}

While early calcium imaging studies have increased our knowledge about cardiac calcium signalling substantially, they have not revealed subcellular detail of the signalling system responsible for excitation-contraction coupling. However, the experimental results discussed in this review make it quite clear that for a detailed understanding of cellular calcium signalling the subcellular spatial features of this system need to be investigated with the highest possible temporal and spatial resolution. With our confocal imaging results we present evidence for the notion that events on a millisecond time scale and on a micrometre spatial scale are functionally important in cardiac musclc cells and most likely also in different cell types and in other signalling systems. In addition, indirect evidence indicates that concentration gradients on submicroscopic scales may also be crucial. Based on the subcellular and molecular information now increasingly becoming available, it should be possible in the foreseeable future to develop "mechanistic" mathematical models of cardiac calcium signalling and excitation-contraction coupling. Using the ever increasing computer power, such models may simulate processes on the molecular level (that is, single channels and transporters) but also encompass processes as complex as the diffusion of calcium in the cellular space and the three dimensional calcium signalling network of the cardiac cell. After completion such a computer model may be able to reproduce and explain the apparently conflicting experimental results on excitation-contraction coupling outlined earlier in this review. Furthermore, spontaneous calcium release events, and linear, circular, and spiral waves of calcium may be simulated by such a model system.

Provided that elementary calcium release signals become spatially and temporally resolvable, the improved confocal imaging technique will enable us to investigate these elementary events in situ. This approach will open a wide new field of research on subcellular calcium signalling and the new possibilities may have an impact comparable to the resolution of elementary single channel opening events made possible by the patch clamp technique.

We would like to thank $\mathrm{Dr} \mathrm{H}$ P Clamann for his valuable comments on the manuscript, and M Herrenschwand for skilful technical help. This work was supported by Swiss National Science Foundation grants to EN (31-28545.90 and 3100-037417.93) and by the Oetliker Foundation.

Key terms: cxcitation-contraction coupling; cardiac myocytes; calcium signalling; fluorescence imaging; confocal microscopy.

Received 13 September 1994; accepted 24 October 1994. Time for primary review 35 days.

1 Bers DM. Excitation-contraction coupling and cardiac contractile force. Dordrecht: Kluwer Academic Publishers, 1991: $1-258$.

2 Wier WG. Cytoplasmic $\left[\mathrm{Ca}^{2+}\right]$ in mammalian ventricle: dynamic control by cellular processes. Annu Rev Physiol 1990;52: $467-85$.
3 Fleischer S, Inui M. Biochemistry and biophysics of excitationcontraction coupling. Annu Rev Biophys Biophys Chem 1989; 18:333-54.

4 Callewaert G. Excitation-contraction coupling in mammalian cardiac cells. Cardiovasc Res 1992;26:923-32.

5 Fabiato A. Simulated calcium current can both cause calcium loading in and trigger calcium release from the sarcoplasmic reticulum of a skinned canine cardiac Purkinje cell. J Gen Physiol 1985;85:291-320.

6 Wier WG, Cannell MB, Berlin JR, Marban E, Lederer WJ Cellular and subcellular heterogeneity of $\left[\mathrm{Ca}^{2+}\right]_{i}$ in single heart cells revealed by fura-2. Science 1987;235:325-8.

7 Takamatsu T, Wier WG. Calcium waves in mammalian heart: quantification of origin, magnitude, waveform, and velocity. FASEB J 1990;4:1519-25.

8 Williams DA, Delbridge LM, Cody SH, Harris PJ, Morgan TO. Spontaneous and propagated calcium release in isolated cardiac myocytes viewed by confocal microscopy. Am J Physiol 1992; 262:C731 -42

9 Cannell MB, Berlin JR, Lederer WJ. Effect of membrane potential changes on the calcium transient in single rat cardiac muscle cells. Science 1987;238:1419-23.

10 DuBell WH, Houser SR. Voltage and beat dependence of $\mathrm{Ca}^{2+}$ transient in feline ventricular myocytes. Am $J$ Physiol 1989; 257:H746-59

11 Beuckelmann DJ, Wier WG. Mechanism of release of calcium from sarcoplasmic reticulum of guinea-pig cardiac cells. J Physiol (Lond) 1988;405:233-55

12 López-Lopéz JR, Shacklock PS, Balke CW, Wier WG. Local, stochastic release of $\mathrm{Ca}^{2+}$ in voltage-clamped rat heart cells: visualization with confocal microscopy. $J$ Physiol (Lond) 1994 480:21-9.

13 Wier WG, Egan TM, López-López JR, Balke CW. Local control of excitation-contraction coupling in rat heart cells. I Physiol (Lond) 1994;474:463-71.

14 Talo A, Stern MD, Spurgeon HA, Isenherg G, I akatta EG Sustained subthreshold-for-twitch depolarization in rat single ventricular myocytes causes sustained calcium channel activation and sarcoplasmic reticulum calcium release. J Gen Physiol 1990;96: 1085-103.

15 Cleemann L, Morad M. Role of $\mathrm{Ca}^{2+}$ channel in cardiac excitationcontraction coupling in the rat: evidence from $\mathrm{Ca}^{2+}$ transients and contraction. J Physiol (Lond) 1991;432:283-312.

16 Niggli E, Lederer WJ. Voltage-independent calcium release in heart muscle. Science 1990;250:565-8.

17 O'Neill SC, Mill JG, Eisner DA. Local activation of contraction in isolated rat ventricular myocytes. Am J Physiol 1990;27: C1165-8.

18 Niggli E, Lederer WJ. Real-time confocal microscopy and calcium measurements in heart muscle cells: towards the development of a fluorescence microscope with high temporal and spatial resolution. Cell Calcium 1990:11:121-30.

19 Lipp P, Niggli E. Ratiometric confocal Ca-measurements with visible wavelength indicators in cardiac myocytes. Cell Calcium $1993 ; 14: 359-72$

20 Niggli E, Piston DW, Kirby MS, et al. A confocal laser scanning microscope designed for indicators with ultra-violet excitation wavelengths. Am J Physiol 1994;35:C303-10

21 Lipp $\mathrm{P}$, Niggli $\mathrm{E}$. Modulation of $\mathrm{Ca}^{2+}$ release in cardiac myocytes insight from subcellular release patterns revealed by confocal microscopy. Circ Res 1994;74:979-90.

22 Cohen NM, Lederer WJ. Changes in the calcium current of rat heart ventricular myocytes during development. $J$ Physiol (Lond) 1988;406: 115-46.

23 Cheng H, Lederer WJ, Cannell MB. Calcium sparks - elementary events underlying excitation-contraction coupling in heart muscle. Science 1993:262:740-4.

24 Williams DA. Mechanisms of calcium release and propagation in cardiac cells - do studies with confocal microscopy add to ou understanding? Cell Calcium 1993;14:724-35.

25 Lipp P, Niggli E. Microscopic spiral-waves reveal positive feedback in subcellular calcium signaling. Biophys $J$ 1993;65:2272-76.

26 Jaffe LF. Classes and mechanisms of calcium waves. Cell Calcium 1993:14:736-45.

27 Fast VG, Pertsov AM. Shift and termination of functional reentry in isolated ventricular preparations with quinidine-induced inhomogeneity in refractory period. $J$ Cardiovasc Electrophysio 1992;3:255-65.

28 Lederer WJ, Tsien R. Transient inward current underlying arrhythmogenic effects of cardiotonic steroids in Purkinje fibres. $J$ Physiol (Lond) 1976;263:73-100.

29 Kass RS. Lederer WJ, Tsien RW, Weingart R. Role of calcium ions in transient inward current and aftercontractions induced by strophantidin in cardiac Purkinje fibres. $J$ Physiol (Lond) 1978 281:187-208. 
30 Kort AA, Lakatta EG. Calcium-dependent mechanical oscillations occur spontaneously in unstimulated mammalian cardiac tissues. Circ Res 1984;54:396-404.

31 Trafford AW, O'Neill SC, Eisner DA. Factors affecting the propagation of locally activated systolic $\mathrm{Ca}$ transients in rat ventricular myocytes. Pflügers Arch 1993;425: 181-3.

32 Meissner G. Ryanodine receptor $\mathrm{Ca}^{2+}$ release channels and their regulation by endogenous effectors. Annu Rev Physiol 1994;56: $485-508$.

33 Sitsapesan R, Williams AJ. Regulation of the gating of the sheep cardiac sarcoplasmic reticulum $\mathrm{Ca}^{2+}$-release channel by luminal $\mathrm{Ca}^{2+}$. J Membr Biol 1994;137:215-26.

34 Knudson CM, Stang KK, Moomaw CR, Slaughter CA, Campbell KP. Primary structure and topological analysis of a skeletal muscle-specific junctional sarcoplasmic reticulum glycoprotein (triadin). J Biol Chem 1993;268: 12646-54.

35 Brandt NR, Caswell AH, Carl SA, et al. Detection and localization of triadin in rat ventricular muscle. J Membr Biol 1993;131:219-28.

36 Coronado R, Morrissette J, Sukhareva M, Vaughan DM. Structure and function of ryanodine receptors. Am J Physiol 1994;266: C1485-504.

37 Wang JX, Best PM. Inactivation of the sarcoplasmic reticulum calcium channel by protein kinase. Nature 1992;359:739-41.

38 Herrmann-Frank A, Varsanyi M. Enhancement of $\mathrm{Ca}^{2+}$ release channel acivity by phosphorylation of the skeletal muscle ryanodine receptor. FEBS Lett 1993;332:237-42.

39 Galione A, Lee $\mathrm{HC}$, Busa WB. $\mathrm{Ca}^{2+}$-induced $\mathrm{Ca}^{2+}$ release in sea urchin egg homogenates: modulation cyclic ADP-ribose. Science 1991:253:1143-6.

40 Meszaros LG, Bak J, Chu A. Cyclic ADP-ribose as an endogenous regulator of the non-skeletal type ryanodine recepor $\mathrm{Ca}^{2+}$ channel. Nature 1993;364:76-9.

41 Vorherr T, Chiesi M, Schwaller R, Carafoli E. Regulation of the calcium ion pump of sarcoplasmic reticulum - Reversible inhibition by phospholamban and by the calmodulin binding domain of the plasma membrane calcium ion pump. Biochemistry 1992;31:371-6.

42 Györke S, Palade P. Role of local $\mathrm{Ca}^{2+}$ domains in activation of $\mathrm{Ca}^{2+}$-induced $\mathrm{Ca}^{2+}$ release in crayfish muscle fibers. Am J Physiol 1993:264:C1505-12.

43 Cannell MB, Cheng H, Lederer WJ. Nifedipine decreases the spatial uniformity of the depolarization-evoked $\mathrm{Ca}^{2+}$ transient in isolated rat cardiac myocytes. (Abstract) J Physiol (Lond) 1994; 447:25P.

44 Stern MD. Theory of excitation-contraction coupling in cardiac muscle. Biophys $J$ 1992;63:497-517.

45 Györke S, Fill M. Ryanodine receptor adaptation-control mechanism of $\mathrm{Ca}^{2+}$-induced $\mathrm{Ca}^{2+}$ release in heart. Science 1993;260: $807-9$.
46 Fabiato A. Time and calcium dependence of activation and inactivation of calcium-induced release of calcium from the sarcoplasmic reticulum of a skinned canine cardiac Purkinje cell. $J$ Gen Physiol 1985;85:247-89.

47 Post JA, Kuwata JH, Langer GA. A discrete $\mathrm{Na}^{+} / \mathrm{Ca}^{2+}$ exchange dependent, $\mathrm{Ca}^{2+}$ compartment in cultured neonatal rat heart cells - characteristics, localization and possible physiological function. Cell Calcium 1993;14:61-71.

48 Leblanc N, Hume JR. Sodium current-induced release of calcium from cardiac sarcoplasmic reticulum. Science 1990;248: $372-6$.

49 Lederer WJ, Niggli E, Hadley RW. Sodium-calcium exchange in excitable cells: fuzzy space. Science 1990;248:283.

50 Niggli E, Lipp P. Subcellular restricted spaces - significance for cell signalling and excitation-contraction coupling. $J$ Muscle Res Cell Motil 1993;14:288-91.

51 Lipp P, Niggli E. Sodium current-induced calcium signals in isolated guinea-pig ventricular myocytes. J Physiol (Lond) 1994: 474:439-46.

52 Rizzuto R, Simpson AWM, Brini M, Pozzan T. Rapid changes of mitochondrial $\mathrm{Ca}^{2+}$ revealed by specifically targeted recombinant aequorin. Nature 1992:358:325-7.

53 Etter EF, Kuhn MA, Fay FS. Detection of changes in nearmembrane $\mathrm{Ca}^{2+}$ concentration using a novel membrane-associated $\mathrm{Ca}^{2+}$ indicator. I Biol Chem 1994:269:10141-9

54 Blatter LA, Wier WG. Agonist-induced $\left[\mathrm{Ca}^{2+}\right]_{i}$ waves and $\mathrm{Ca}^{2+}$. induced $\mathrm{Ca}^{2+}$ release in mammalian vascular smooth muscle cells. Am J Physiol 1992;263:H576-86.

55 Cornell-Bell AH, Finkbeiner SM, Cooper MS, Smith SJ Glutamate induces calcium waves in cultured astrocytes: longrange glial signaling. Science 1990;247:470-3.

56 Somogyi R, Stucki JW. Hormone-induced calcium oscillations in liver cells can be explained by a simple one pool model. $J$ Biol Chem 1991;266:11068-77.

57 Woods NM, Cuthbertson KSR, Cobbold PH. Agonist induced oscillations in cytoplasmic free calcium concentration in single rat hepatocytes. Cell Calcium 1987;8:79-100.

58 Lechleiter L, Girard S, Peralta E, Clapham D. Spiral calcium wave propagation and annihilation in Xenopus laevis oocytes. Science 1991:252:123-6.

59 Parker I, Ivorra I. Localized all-or-none calcium liberation by inositol trisphosphate. Science 1990;250:977-9.

60 Camacho P, Lechleiter JD. Increased frequency of calcium waves in Xenopus laevis oocytes that express a calcium-ATPase. Science 1993:260:226-9.

61 Girard S, Clapham D. Acceleration of intracellular calcium waves in Xenopus-oocytes by calcium influx. Science 1993;260: $229-32$

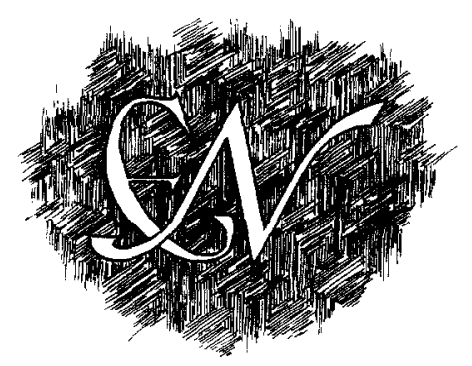

